\title{
INVESTIGACIONES PARA EL TRATAMIENTO SILVICOLA Y MANEJO DE RENOVALES DE Nothofagus EN LA ZONA CENTRO DE CHILE'
}

\author{
BERNARDO AVILÉS
}

Ingeniero Forestal, Facultad de Ciencias Forestales Universidad de Concepción, Dr. Wolframstr 5 , Würzburg, Alemania

\section{RESUMEN}

En renovales de Nothofagus ubicados en la precondillera andina de la LX Región se prueban $y$ evaluan intervenciones de raleo selectivo en rodales coetáneos, bietáneos y multietáneos, originados en diferentes tipos $e$ intensidades de utilización anterior.

Se aplican raleos selectivos y se seleccionan árboles de cosecha, se evaluan los resultados económicamente y se concluye que el uso de métodos silvicolas adecuados y la maximización de la calidad y utilización de la madera, con el apoyo de normas de clasificación, son prácticas que pueden resultar exitosas en renovales de diferentes estructuras.

Palahras Clave: Silvicultura, Renovales. Nothufagus

\section{ABSTRACT}

In second growth Nothofagus forests, located near the Andean Ranges of the LX Region of the country, selective thinnings are tried in stands of even age, two equal ages and multiple ages, originated in different types of previous utilization.

Cuts are applied and trees to harvest are selected. Results are economically evaluated and it is concluded that adecuated silvicultural techniques to maximice the wood quality and utilization, with the help of clasification norms, can be succesfully used under different structured stands.

Keywords: Silviculture, Second (irouth Forests, Nothofagus

\footnotetext{
1 Imvestigación dirigida por el Profesor, Dr. Jürgen Huss, Director del Instituto de Silvicultura de la Universidad de Freiburg 79085 Freiburg. Alemania.
} 


\section{INTRODUCCION}

Renovales son bosques secundarios que se originaron de la explotación selectiva, talas rasas y roces a fuego de los bosques primarios mediante regeneración natural por semillas o por tocones. Estos renovales se encuentran especialmente entre la VII región ( $35^{\circ}$ latitud sur) y la $\mathrm{X}$ región ( $41^{\circ}$ latitud sur), tanto en la precordillera de los Andes como en la cordillera de la Costa (Puente et. al., 1981; Donoso, 1981). Su edad es variable y es posible encontrar rodales con edades entre 20 y 100 años, especialmente de las especies Nothofagus alpina, Nothofagus obliqua y Nothofagus dombeyi. La superficie que ocupan estos renovales no ha sido determinada. Sin embargo, se cree que se trataria de unas 600.000 ha (De Camino et. al., 1974; Yoma, 1984; Ulloa, 1984). Las dificultades para determinar la superficie potencial de renovales se debe fundamentalmente a las diferentes definiciones existentes del termino "Renoval", en muchos casos subjetivas y que no definen su potencial (INFOR, 1967; Reglamento 2565/DL. 701, 1979; Donoso, 1981), y a la inexistencia de inventarios y catastros de éste recurso.

En los últimos años se han mejorado notablemente las posibilidades de utilización y comercialización de las especies que componen los bosques de renovales. La demanda por estos productos ha ocasionado que los propietarios forestales tiendan a una utilización rápida de éste recurso con el fin de obtener altos beneficios económicos en el corto plazo y que se contraponen a las necesidades sociales, que se caracterizan por favorecer la mantención y acrecentamiento del recurso prefiriendo largas rotaciones. Esta utilización ha tenido la característica de explotaciones a tala rasa, sucesivas, o bien a cortas selectivas que reducen el valor del bosque en algunos años y destruyen los beneficios ambientales producidos por el éste, como son la protección de los cursos de agua y del suelo, además. del hábitat para la fauna y la flora nativa, en algunos casos única y de gran valor para Chile.

\section{OBJETIVOS}

El objetivo de ésta investigación es mostrar modelos conceptuales de como los renovales de Nothofagus en forma planificada y sostenida pueden ser transformados en bosques comerciales de alta productividad, sin alterar sus funciones ecológicas. Para ello se han considerado los aspectos silvicolas, de la calidad de la madera y sus usos y los aspectos económicos, en general. Mediante la transformación de estos bosques se intenta modificar la estructura del rodal en tal magnitud que sea posible la producción de madera de alta calidad. Los resultados de ésta investigación deben además, entregar conclusiones respecto de las posibilidades de clasificación de estos renovales, de la posibilidad de practicar raleos selectivos. con definición de árboles cosecha, y mostrar y verificar la aptitud maderera de las especies $\mathbf{N}$. alpina, N. obliqua y $\mathbf{N}$. dombeyi para 
la producción de madera de alta calidad y valor comercial. Finalmente, se espera obtener resultados de carácter monetario en forma de modelos realizables, con los cuales se pretendió hacer un aporte al estudio de los bosques de renovales en Chile.

\section{ÁREA dE ESTUDIO}

Las investigaciones se realizaron en la Hacienda Jauja, Provincia de Malleco en la IX Región, perteneciente a la Empresa Forestal Mininco S.A.

El área investigada se caracteriza por tener un clima mediterráneo con abundante precipitación en las estaciones de otoño e invierno y períodos secos en verano. La humedad relativa del aire, con valores cercanos al $70 \%$, favorece el crecimiento de bosques en la zona. El suelo en su mayor proporción corresponde a depositaciones de cenizas de origen volcánico. Estos suelos son característicos en la zona central de Chile y son denominados "Trumaos ". Ellos constituyen los suelos de mayor potencial para la producción. En general, son suelos profundos y de estructura relativamente suelta, sin embargo, presentan tendencia a la compactación. Su fertilidad es alta, poseen un alto porcentaje de materia orgánica, alta capacidad de almacenamiento de agua y un pH entre 5 y 6: el fósforo es fijado en una alta proporción.

\section{METODOLOGÍA}

\section{Clasificación de los Rodales Investigados}

La comprobación de las clasificaciones de renovales existentes mostró que estos tienen una distribución espacial irregular y que no es posible llegar a una caracterización y estratificación mediante área basal, número de árboles por hectárea y diámetro promedio. Las formas de clasificación utilizadas no aportan la información base necesaria para establecer intervenciones silvicolas con el fin de una producción de madera de alta calidad, en forma sostenida y permanente (Herrera y May, 1975, 1976; Wadsworth, 1976; Puente et. al., 1979; Donoso, 1981; Nuñez y Peñaloza, 1985; Espinosa, 1990; Pokorny, 1991). El resultado de la estratificación realizada en base a fotointerpretación. control de terreno y muestreo de rodales modales permite identificar tres tipos de rodales básicos: Coetáneos, Bietáneos y Multietáneos. Estos tipos característicos tienen su origen en el uso al que fueron sujetos en el pasado, entre los cuales se distinguen:

- Uso por Superficie: Durante la colonización europea se explotaron bosques para habilitar terrenos para la agricultura y la ganadería. En muchos casos y debido a la productividad del suelo, muchas de estas áreas fueron abandonadas, lo que más adelante generó bosques homogéneos provenientes de regeneración vegetativa $\mathbf{y}$ de semillas. Una parte de los rodales investigados fue raleada por lo bajo con diferente intensidad. de modo que fue posible reconocer dos formas: 
Rodales raleados compuestos principalmente por la especie $\mathbf{N}$. alpina. Debido a las intervenciones silvícolas no existen otros estratos. En general, el desarrollo de copas es limitado debido a la fuerte competencia entre individuos a ese nivel. La distribución diamétrica muestra una gran dispersión, en donde los individuos de mayor diámetro se caracterizan por tener mala forma y abundantes ramas.

Rodales no intervenidos que se sometieron a uso por superficie, presentan dos estratos bien marcados y se caracterizan por ser más jóvenes. La especie de mayor abundancia es N. alpina, la que está representada por árboles de buena calidad aptos para la producción de madera de buen valor.

- Uso Selectivo: El uso selectivo, en el cual se extrajeron sólo maderas valiosas, condujo a estos rodales a una marcada estructura de estratos. Desde el comienzo de la colonización española en Chile ha sido relevante este tipo de uso, en donde se extraen los mejores individuos en calidad maderera, mientras que aquellos de mala forma y sin valor comercial permanecen en el bosque y posteriormente alcanzan los estratos superiores. Los árboles, resultado de este tipo de intervención, muestran en general copas grandes, curvaturas de fuste y abundancia de ramas. Los claros generados por la explotación sirvieron para el establecimiento de otras especies, diferentes de Nothofagus, que ocupan parte del segundo estrato. El tercer estrato se compone, especialmente, de la especie $\mathbf{N}$. alpina, proveniente de regeneración natural de tocones y de semillas. En rodales sujetos a este tipo de uso resulta típica una estructura de mosaico y una distribución espacial de bosquetes homogéneos de diferente tamaño y forma. Además, es característico que estos bosques presentan árboles de gran tamaño, sobremaduros y enfermos, árboles solitarios de buen crecimiento y calidad maderera y grupos de regeneración natural.

\section{Raleo Selectivo y Selección De Árboles Cosecha}

Burschel y Huss (1987) aseguran que: “...junto al raleo selectivo se debe intervenir, fuertemente, y liberar en lo posible aquellos árboles del estrato dominante, de mayor vitalidad, mejores características cualitativas y mayor valor comercial o árboles cosecha, los cuales serán fomentados en su desarrollo. En las intervenciones siguientes se debe intervenir a nivel de copas, aclareos para el crecimiento de la luz, con lo que se logrará la expansión y formación de la copa, que posteriormente inducirá un aumento en el crecimiento en diámetro. En los estratos suprimidos, medio en algunos casos y suprimido en todos los casos, no se intervendrá, ya que ellos tienen como función la protección del suelo y de los fustes".

En Chile, en forma tradicional a partir de 1960 , se han realizado raleos por lo bajo y sus variantes más conservadoras para la intervención de los bosques de renovales (Rocuant, 1969; Puente y Herrera. 1978; Puente. 1979, 1983; Puente et. al., 1981; Nuñez y Peñaloza. 1985). Las experiencias realizadas, según la documentación 
existente, no han considerado otras variantes, como raleos por lo alto, selectivos con y sin marcación de árboles cosecha y aclareos para promover el crecimiento de luz. Excepciones son los trabajos de Grosse (1990) y Csapek (1992), que han probado algunas de éstas variantes.

El procedimiento raleos selectivos y selección de árboles cosecha, fue elegido debido a que ha sido extensamente reconocido en Europa Central para la producción de madera de alta calidad, como una alternativa viable a desarrollar en bosques naturales (Schädelin, 1942; Assmann, 1961; Leibundgut, 1966; Altherr, 1974; Abetz, 1979; Köpsell, 1983; Burschel y Huss, 1987). El número de árboles cosecha se calculó en base a el área basal máxima potencial que es posible encontrar en un renoval puro compuesto especialmente de N. alpina; este procedimiento fue desarrollado por Abetz (1979) como una forma de obtener lineamientos generales para las intervenciones silvícolas en Fagus sylvatica. Para rodales con un área basal entre 35 y $50 \mathrm{~m}^{2} / \mathrm{ha}$ y un diámetro objetivo a la cosecha de $60 \mathrm{~cm}$, son necesarios entre 125 y 200 árboles cosecha/ha. El diámetro objetivo se consideró debido a la posibilidad de obtener el mayor rendimiento económico del bosque, al aumento creciente de los rendimientos en la transformación mecánica y a la alternativa de competir con maderas tropicales de Africa y Asia y latifoliadas europeas.

De los raleos realizados en los tres tipos de renovales intervenidos se resumen los siguientes resultados:

En los rodales coetáneos (rodales adultos homogéneos) la selección de árboles cosecha no presenta problemas de carácter técnico. Es posible encontrar una cantidad considerable de árboles bien formados, que presentan excelentes características de fuste. Una gran ventaja que se presenta en este tipo de rodales es que a través de la intervención se benefician otros individuos, que no necesariamente formaran parte del rodal final, pero que aumentaran considerablemente el volumen de las cortas intermedias, aumentando el rendimiento final del sitio. Sin embargo, en aquellos rodales que fueron raleados en años anteriores y en donde se eliminaron completamente los estratos secundarios, existe la posibilidad de que a causa del raleo se produzcan epicormios que tendrán como resultado una pérdida en la calidad de la madera.

En rodales bietáneos (rodales de dos estratos, jóvenes y homogéneos) fue posible encontrar en gran cantidad árboles con buenas características. La selección no presenta dificultades. Sin embargo, parece ser demasiado temprano para realizar una selección definitiva de árboles cosecha. En estos rodales y debido a su edad, cerca de 30 años, existen las mejores posibilidades de producir madera de calidad en el corto plazo ya que se espera una rápida respuesta a la intervención. En este caso se intervino solo alrededor del árbol, eliminando los competidores directos. Otros raleos serán necesarios en los próximos años, en los cuales se deberá seleccionar en forma definitiva los árboles cosecha. La selección posterior y los raleos deberán ser orientados a mantener el crecimiento y calidad de estos árboles. 
En rodales multietáneos (rodales con más de tres estratos heterogéneos y provenientes de uso selectivo) la selección de árboles cosecha resulta técnicamente muy difícil. En estos rodales no es posible formular en primera instancia, en forma clara y definitiva, un concepto de tratamiento silvicola. La forma de mosaico que presentan estos rodales, en los cuales es posible reconocer más de una estructura, que generalmente presenta agrupaciones de árboles de diferentes especies, tamaños, calidades y dominancia, no permite aplicar un concepto que lleve a la homogeneidad de los rodales y a la intervención por superficie. Parece adecuado en este caso, en primer lugar la eliminación de los árboles dominantes pertenecientes a la especie $\mathbf{N}$. dombeyi, que se caracterizan por su gran tamaño y reducida calidad. La segunda etapa deberá considerar la realización de raleos frecuentes destinados a liberar los árboles de buena forma y crecimiento que se encuentran creciendo bajo las copas de estos árboles sobremaduros y que en algunos casos se encuentra prácticamente suprimidos. Finalmente, será necesario en estos rodales realizar más de una actividad silvícola, i.e. raleo, selección, liberación de árboles, regeneración de especies económicamente atractivas como es el caso de $\mathrm{N}$. alpina. La flexibilidad y el buen criterio en el tratamiento a realizar son la base del éxito en la transformación de este tipo, que es abundante y generalizado en la zona de estudio.

\section{Calidad de Árboles en Pie}

La determinación de la calidad en pie y en trozas es fundamental para tomar decisiones silvicolas: la selección de rodales, la selección de árboles de cosecha, la comercialización de madera y otras actividades exigen un conocimiento pleno de la calidad.

Sin embargo, se debe considerar que la estimación de la calidad, especialmente en árboles jóvenes, es dificil y sujeta a error por las modificaciones de la calidad a través del tiempo Arnswalt (1950). Schulz (1958, 1959, 1961).

En esta parte se investigó si los árboles seleccionados son aptos para la producción de madera de alto valor comercial. La caracterización y evaluación de los árboles en pie resultó ser muy difícil de evaluar en términos estadísticos, especialmente debido a la alta variabilidad y dispersión de las variables analizadas, muchas de las cuales son generadas por los factores ecológicos y el ambiente generado en cada rodal. Sin embargo, desde el punto de vista visual, la caracterización es una ayuda para la selección de árboles. así como para la ejecución de los raleos es una herramienta de gran valor que debe ser positivamente valorada y utilizada.

El análisis de las características de la corteza no entregó diferencias significativas en relación a la dimensión, entre los rodales tipos. Estas características de la corteza se relacionan con el $d_{1,3}$ y no se manifiestan en árboles con $d_{1,3}$ superior a $30 \mathrm{~cm}$. La selección de los árboles cosecha debe, en todo caso, orientarse de acuerdo a la siguiente serie de características: Ramas secas ubicadas en los primeros $10 \mathrm{~m}$ del fuste; 
cicatrizaciones de ramas que muestren un proceso lento de recubrimiento, como indicador de pudrición interior; protuberancias, bigotes de chino con ángulos agudos y enfermedades de la corteza.

Los resultados indican que existe una secuencia cronológica en el proceso de cicatrización de ramas; rama verde, rama seca, muñón, protuberancia, bigote de chino. Además, de agallas, rosetas, estrellas y perforaciones varias. Finalmente, se debe destacar que la especie $\mathbf{N}$. alpina presenta una gran capacidad y rapidez para la cicatrización, que es independiente de las condiciones ecológicas del rodal, en especial de la cantidad de luz difusa y directa que esta presente en el rodal.

\section{Calidad de Trozas y Madera Aserrada en Bloques}

El objetivo de esta investigación, resumida en dos partes, fue probar y aclarar de acuerdo a métodos establecidos y de validez general en que medida las características de la corteza afectan y caracterizan la calidad de la madera. Además, de probar la aptitud de las especies de Nothofagus para producir madera de calidad en una proporción tal que sea posible competir con esta materia prima en mercados internacionales. Las características cualitativas de la madera fueron evaluadas mediante la Norma HKS (Handelsklassensortierung) para madera en trozas y Norma DIN-68369 para madera aserrada en bloques. Las clasificaciones entregaron los siguientes resultados:

\section{Clasificación Norma HKS ${ }^{2}$}

Las ramas secas bajo $4 \mathrm{~m}$ de altura y con diámetro sobre $6 \mathrm{~cm}$ son relevante e indicadoras de pudrición. Las grietas, independiente de su forma y tamaño, son relevantes. Se recomienda uso de sellantes o corchetes metálicos. Se recomienda restringir el volteo y trozado a los períodos de otono e invierno.

Las pudriciones, en trozas, tienen poca frecuencia en las especies $\mathbf{N}$. alpina y $\mathbf{N}$. obliqua. Mientras, que en N. dombeyi estas son muy frecuentes y desvalorizan considerablemente la madera.

La clasificación según HKS, sin considerar grietas, fue de $7 \%$ en volumen en clase A $80 \%$ en clase B y $13 \%$ en clase C. El rendimiento en madera rolliza según HKS fue de $61 \%$. En N. dombeyi este valor se reduce a $40 \%$ por efecto de pudriciones.

La curvatura no constituye en las especies investigadas un factor cualitativo importante; $95 \%$ de los trozos presenta valores menores de $2 \mathrm{~cm} / \mathrm{m}$ para un valor crítico, según $H K S$, de $5 \mathrm{~cm} / \mathrm{m}$. La heterogeneidad del ancho de los anillos de crecimiento producida por falta de intervención silvicola limita la posibilidad de uso de

2 Bundesmınistenum Fur Emahrung. Landwirtschaft und Forsten (1969): Handelsklassensortıenung 
la madera para chapa. La duraminización es dependiente del $d_{1,3}$ y es un factor favorable desde el punto de vista estético. La norma HKS, con modificaciones, puede ser utilizada sin restricción.

\section{Clasificación DIN-68369}

Los resultados indican que el $50 \%$ de las piezas aserradas presenta ancho de anillos regular y menor de $1,5 \mathrm{~mm}$. El $60 \%$ presenta algún tipo de deformación en donde la arqueadura representa el $80 \%$. El $65 \%$ de las piezas presenta duramen y excelente aspecto. La grieta y la acebolladura son importantes para la clasificación y se estima que el $\mathbf{8 0} \%$ de las piezas la presenta. La pudrición café predomina sobre la pudrición blanca, especialmente, en N. alpina. El $\mathbf{8 0 \%}$ de la pudrición se encuentra en estado inicial.

N. alpina no presenta daños por insectos mientras que N. dombeyi se encuentra dañada en un $50 \%$ de los casos. Además, se encontró que el $15 \%$ del daño es superficial y el $\mathbf{8 0} \%$ profundo en el cuerpo de la madera y que abarca el duramen.

N. alpina presenta la mayor abundancia de nudos, sin embargo el $75 \%$ estaban sanos. Los nudos, en otras especies, son un aspecto secundario para la clasificación.

La clasificación DIN-68369 entrego menos de $20 \%$ en la clase I, cerca de $50 \%$ en la clase II y menos de $30 \%$ en la clase III. La norma es posible de aplicar sin problema en las especies analizadas.

\section{Crecimiento en Diametro y Prognosis del Crecimiento}

Otro objetivo de la investigación fue analizar algunas variables de crecimiento en la especie $\mathbf{N}$. alpina, especialmente el $d_{1,3}$ y en árboles dominantes, para poder determinar el crecimiento actual y de esa forma proyectar el crecimiento de los árboles cosecha.

La investigación indica que en los árboles seleccionados el crecimiento en diámetro, para una situación sin intervención, oscila entre 0,43 y $0,52 \mathrm{~cm} /$ año. Las variaciones en crecimiento presentan coeficientes de variación promedio de hasta $38 \%$. El crecimiento en diámetro se mostró independiente del $\mathrm{d}_{1,3}$. Una comparación con los resultados de investigaciones existentes no fue posible debido a que el objeto estudiado es diferente; en esta investigación se midió y analizó sólo árboles dominantes en cada estrato.

La prognosis de crecimiento de los árboles cosecha se realizó en base al crecimiento promedio observado en las mediciones, para lo cual fue posible considerar tres alternativas optimista, media y pesimista. El ajuste de funciones de regresión múltiple, en las cuales se consideró el $d_{1,3}$ y la edad de los árboles, entregó resultados insuficientes para efectuar una provección confiable. Se observa que a medida que el 
diámetro aumenta se produce un aumento mas que proporcional del crecimiento. La inclusión de la edad actúa en forma contraria, es decir a mayor edad menor crecimiento. Sin embargo, en la investigación ya había sido probado que los árboles de N. alpina, aún en edad avanzada crecen a tasas similares que árboles jóvenes. Además, y debido a la longevidad de la especie, se cree que esta pueda reaccionar a las intervenciones aún en edades avanzadas; hecho que debe ser probado ya que es de gran importancia para la intervenir.

\section{Estructura de Costos y Cálculo Monetario}

El objetivo de esta parte fue recopilar información básica sobre la estructura y característica de costos que se originan en un primer raleo. Esta información tiene, sin embargo, el carácter de preliminar, ya que un aumento de la escala de operaciones podria influir en forma significativa. Los resultados más importantes se resumen a continuación:

Los costos de selección de árboles cosecha, marcación, construcción de calles de madereo, volteo, trozado, preparación de la madera incluyendo clasificación y transporte a la planta de Nacimiento alcanzaron a $17 \mathrm{US} \$ \mathrm{~m}^{3}$, para madera en trozas y $19 \mathrm{US} / \mathrm{m}^{3}$ para madera de pulpa y leña. El mayor costo de la producción de madera de pulpa y leña se explica por el arrastre y arrumado de trozas. Los costos determinados se distribuyen en partes homogéneas respecto del trabajo manual y mecanizado.

Los costos de transporte alcanzaron a un $65 \%$ del costo total; el resto de las actividades cubrió el $35 \%$, que se considera bajo. Como se indicó, el costo total puede experimentar variaciones si se aumenta la fuerza de trabajo, el control y la infraestnuctura. Una compensación del aumento de los costos puede ser alcanzada mediante operaciones de escala, aumento de la eficiencia y control de las inversiones. Si las operaciones abarcan grandes superficies puede aumentar el volumen de madera aprovechable en las clases de mayor valor, aumentando la diferencia entre costo de producción y precio de venta.

El cálculo de los costos límites realizado para el primer tratamiento entregó ganancias de 2.000 US\$/ha en los rodales coetáneos (un estrato), 740 US\$/ha en los rodales Bietáneos (dos estratos) y 1.200 US\$/ha en rodales multietáneos (tres estratos y más).

El precio de venta de la madera de trozas cubre un $60 \%$ de los costos de producción y $32 \%$ de la madera para pulpa. Los costos de producción de leña no se cubren con el precio de venta y generan un déficit en la preparación de la madera de alrededor de un $36 \%$.

Un cálculo monetario de la producción, es decir sin considerar una tasa de interés alternativa, ya que se parte de la base de que todas las intervenciones se financian, en cada uno de los rodales analizados y al final de la rotación entrega ingresos entre $\mathbf{4 0 . 0 0 0}$ y 60.000 US\$/ha. 
Estos resultados, provenientes de modelos silviculturales y de clasificación y aprovechamiento de la madera, permiten concluir que el tratamiento silvícola y el manejo de los renovales de Nothofagus orientados a la producción de madera de buena calidad es factible podría ser una alternativa viable que conduciria a éxitos en los resultados monetarios y financieros.

\section{CONCLUSIONES}

Como resultado general de este conjunto de investigaciones es posible concluir que la aplicación de métodos silvícolas adecuados y la maximización de la calidad y uso de la madera, apoyadas en normas de clasificación, pueden ser aplicados en bosques de renovales de diferentes estructuras en forma exitosa. En el caso de no ser así, y es probable que en muchos casos ocurra, el manejo de los renovales debería ser enfrentado mediante una subvención estatal, la que debería considerar a lo menos:

Disminuir en el mediano plazo la diferencia entre la valoración privada y estatal de este recurso y asi evitar usos del bosque y del suelo que, desde el punto de vista social, no son óptimos,

Que la asignación de recursos sea hecha en forma tal que priorice ubicaciones geográficas, grupos de especies, estructura de bosques y aumente el valor económico de estos. especialmente respecto de las plantaciones. Asimismo se debe tender a valorar y maximizar las externalidades que están siendo producidas por estos bosques,

Finalmente, es una realidad tangible que existe la urgente necesidad de la mantención de los bosques de renovales de Nothofagus, debido que desde el punto de vista biológico representan un recurso de alto valor, especialmente si se considera que estos protegen fuentes de agua y mantienen su cantidad y calidad, vital para el crecimiento industrial, y que, además, son hábitat para flora y fauna nativa del pais.

\section{AGRADECIMIENTOS}

El Autor agradece al Consejo Alemán de Intercambio Académico (=DAAD) y al Instituto de Silvicultura de la Universidad de Freiburg, Alemania, que hicieron posible la realización de esta investigación. Asimismo agradece a la Empresa Forestal Mininco que puso a disposición los bosques de renovales de la Hacienda Jauja. Un reconocimiento especial al Profesor Dr. Jürgen Huss, por su dedicación y optimismo respecto del tema Renovales.

\section{REFERENCIAS BIBLIOGRAFICAS}

Abetz, P., 1979. Zum Konzept einer Z. - Baum - orientierten kontrollmethode. Allgemeine Forstund J. Ztg. 151:65-95. 
Altherr, E., 1974. Vorschläge für die Durchforstung von Buchenbestânden. (Aus der Forstlichen Versuchs - und Forschungsanstalt Baden - Wurttemberg. - Abt. Waldwachstum, Freiburg i.Br.) 19 pp.

Arnswaldt, H.J.V., 1962. Wertkontrolle und Betriebswirtschaft. FA. 161 pp.

Assmann, E., 1961. Waldertragskunde. München, Bonn, Wien. 491 pp.

Burschel, P. y J. Huss., 1987. Grundriss des Waldbaus. Ein Leitfaden für Studium und Praxis. Hamburg und Berlin: Parey. 352 pp.

Corporación Nacional Forestal, 1981. Reglamento del Decreto Ley 701/1974 sobre Fomento Forestal en Chile. Chile Forestal. Edición Especial. Santiago de Chile.

Csapek, G., 1991. Zur Durchforstung von Nothofagussekundărwăldern in Mittelchile. Tesis Forstwissencshaftliche Fakultăt Universităt Freiburg. 75 pp.

De Camino, R., Smith, B., Benavides, M., y Rodas, J., 1974. Los Renovales de Bosque Nativo como Recurso Forestal. Antecedentes para la Discusión del Problema. Instituto de Manejo Forestal y Economia Forestal. UACH. Charlas y Conferencias (2): 19-33.

Donoso, C., 1981. Tipos Forestales de los Bosques Nativos de Chile. Investigación y Desarrollo Forestal. FO:DP/CHI/76/003. Santiago de Chile. Documento de Trabajo No38. 70 pp.

Espinosa, M., 1990. Rodalización y Determinación de Opciones de Manejo Silvícola para los Rodales no Manejados de Jauja y Santa Luisa. FORVESA. Chillán-Chile. 81 pp.

Grosse, H., 1990. Antecedentes para el Manejo de Renovales de Rauli. Corma (1):16-20.

Herrera, J. y May, F. ,1975. Proyecto de Raleos en Jauja. FORVESA: Informe $\mathrm{N}^{\circ} 19 \mathrm{~S}$.

Herrera, J. y May, F., 1976. Proyecto de Raleos en Jauja. FORVESA: Informe N2. 15

Instituto Forestal, 1967. Clasificación Preliminar del Bosque Nativo en Chile. Informe Técnico N27. Santiago de Chile. Chile.

Koepsell, R., 1983. Charakteristische Kennzifferstrukturen naturgemaesse bewirtschafteter Forsbetribe. Naturgemaesse Waldwirtschaft. Sonderdruck Forstarchiv (2, 3): 16-22.

Leibundgut, H., 1966. Die Waldpflege. Verlag Paul Haupt Bern und Stuttgart. 192 pp.

Nuñez, P. y P. Peñaloza, 1985. Estudio de Raleo y otras Técnicas para el Manejo de los Renovales de Raulí (Nothofagus alpina) y Roble (Nothofagus obliqua). Informe Convenio 80. Fac. de Cs. For. UACH. Proyecto CONAF/PNUD/FAO-CHI/76/003. Santiago de Chile. $109 \mathrm{~S}$.

Pokorny, B., 1991. Eignung der von der Baumart Nothofagus alpina Geprägten Mittelchilenischen Renovalesbestände für eine Wertholzorientierte Bewirtschaftung. Tesis, Waldbau Institut d. Universität Freiburg. $98 \mathrm{pp}$.

Puente, M., 1979. Control de Raleos en Renovales de Rauli del Fundo Jauja. Industrias Forestales S.A. Facultad de Ingenieria Forestal. Universidad Austral de Chile. Valdivia, Chile. $38 \mathrm{pp}$. 
Puente, M. y Herrera, J., 1978. Informe Sobre el Raleo de Renovales en el Fundo Jauja. Industrias Forestales S.A. Nacimiento, Chile. 11 pp.

Puente, M., Donoso. C., Peñaloza, R. y Morales, E., 1979. Manejo de Renovales de Raulí y Roble Identificación y Caracterización de Renovales de Raulí y Roble. INVESTIGACION Y DESARROLLO FORESTAL: Dcto. de Trabajo N29. FO: DP/CHI/76/003. Santiago de Chile. $84 \mathrm{~S}$.

Puente, M., Donoso, C., Peñaloza, R., Paredes, R., Morales, R. y Engdahl, O., 1981. Estudio de Raleo y otras Técnicas para el Manejo de Renovales de Rauli y Roble. Instalación de Ensayos de Raleo. Investigación y Desarrollo Forestal: Documento de trabajo $\mathrm{N} \times 41$. FO: $\mathrm{DP} / \mathrm{CHI} / 76 / 003$. Santiago de Chile. $74 \mathrm{pp}$.

Puente, M., 1983. Control de Raleos en Renovales de Raulí del Fundo Jauja. Nacimiento, Chile. $29 \mathrm{pp}$.

Rocuant, L, 1969. Raleos en Renovales de Rauli-Roble en la Cordillera de Nahuelbuta. Circular Informativa N24. Universidad de Concepción. Chillán, Chile. 22 pp.

Schädelin, W., 1942. Die Auslesedurchforstung als Erziehungsbetrieb hőchter Wertleistung. 3. neu durchgearbeitete Auflage. Bern/Leipzig: Verlag Paul Haupt. $174 \mathrm{pp}$.

Schulz, H., 1958. Merkmale überwachsender Hozfehler in der Baumrinde. Holz-Zentralblatt (71): $919-920$.

Schulz, H., 1959. Güteklassen des Stammholzes und ihre Abgrenzung gegeneinander. HolzZentralblatt 85: 753-757.

Schulz, H., 1961. Die Beurteilung der Qualitätsentwicklung junger Băume. Forstarchiv 32: 8999.

Speidel, G., 1978. Forstliche Betriebswirtschaftslehre. Berlin. Parey. 226 pp.

Ulloa, F., 1984. Alcances sobre la Disponibilidad de Bosque Nativo Comercial en Chile. Actas XI Jornadas Forestales. Concepción, Chile. 4: 65-70.

Wadsworth, R. K. 1976. Aspectos Ecológicos y Crecimiento del Rauli y sus Asociados en Bosques de Segundo Crecimiento en las Provincias de Bio-Bio, Malleco y Cautin, Chile. Boletin Técnico N 37 . Facultad de Ciencias Forestales, Universidad de Chile. Santiago de Chile, 47 pp.

Yoma, R., 1984. Ideas Preliminares para un Proyecto de Bonificación por Manejo de Renovales y por Enriquecimiento del Bosque Nativo. Actas XI Jornadas Forestales. Concepción, Chile. pp $38-41$. 